\title{
Dolor Músculo-Esquelético y su Asociación con Factores de Riesgo Ergonómicos, en Trabajadores Administrativos
}

\author{
Paola Vernaza-Pinzón ${ }^{1}$ y Carlos H. Sierra-Torres ${ }^{2}$ \\ ${ }^{1}$ Fisioterapeuta. Especialista en Epidemiología General. Departamento de Fisioterapia. \\ Facultad de Ciencias de la Salud, Universidad del Cauca, Popayán, Colombia. Teléfono: +57- \\ 2-8234118, Ext. 114. E-mail: pvernaza@unicauca.edu.co \\ ${ }^{2}$ Biólogo. Ph.D. Ciencias Biomédicas, Departamento de Ciencias Fisiológicas, Facultad de \\ Ciencias de la Salud, Universidad del Cauca, Popayán, Colombia. Teléfono: 57-2-8209872. \\ E-mail: hsierra@unicauca.edu.co
}

Recibido 31 Mayo 2005/Enviado para Modificación 10 Agosto 2005/Aceptado 27 Septiembre 2005

\section{RESUMEN}

Objetivo Establecer la frecuencia de las lesiones músculo-esqueléticas en trabajadores administrativos y su posible asociación con factores de riesgo ergonómico.

Métodos Se realizo un estudio observacional descriptivo a 145 trabajadores de la Universidad del Cauca en Popayán, Colombia, entre julio 2002 y junio 2003. Para la recolección de la información se utilizaron dos instrumentos: un formato para análisis del puesto de trabajo y un cuestionario para el análisis de síntomas músculo-esqueléticos.

Resultados El $57 \%$ de los trabajadores administrativos presentaron síntomas de dolor. Las Lesiones más frecuentes se encontraron en la zona baja de la espalda (56,6 \%), la zona alta de la espalda $(53,1 \%)$ y el cuello $(49,0$ $\%$ ). Los trabajadores que mostraron con mayor frecuencia la postura inclinado, presentaron un Odds Ratio-OR de 3,0 y los trabajadores que durante su actividad mostraron con mayor frecuencia el caminar, presentaron un OR de 2,8 para la presencia de dolor músculo-esquelético en la zona baja de la espalda.

Conclusiones Los resultados de este estudio revelan que existe una asociación entre la exposición a factores de riesgo biomecánico y la presencia de lesiones músculo-esqueléticas, indicando que posturas de trabajo forzadas significan mayor riesgo. Por lo tanto, este tipo de trastornos podrían llegar a incapacitar al trabajador en las actividades de la vida diaria.

Palabras Clave: Enfermedades musculoesqueléticas, factores de riesgo ergonomía, personal administrativo, Colombia (fuente: DeCS, BIREME). 


\section{Musculoskeletal pain and its association with ergonomic risk factors in administrative workers}

Objective Establishing the frequency of musculoskeletal lesions in administrative workers and its possible association with ergonomic risk factors.

Methods A descriptive study of 145 workers from the Universidad del Cauca in Popayán was carried out between July 2002 and June 2003. Two instruments were used for collecting the information: a format for analysing working posture and a questionnaire for analysing musculoskeletal symptoms.

Results Fifty-seven percent of the administrative workers presented symptoms of pain. The most frequent lesions were found in the lower back $(56,6$ $\%)$, upper back (53,1\%) and the neck (49\%). Workers who sat for most of their working day presented an OR of 3,0 for lower back lesions and workers who had to walk more during their daily activity presented an OR of 2,8.

Conclusions The results of this study reveal an association between exposure to biomechanical risk factors and the presence of musculoskeletal lesion, indicating that forced work postures signify greater risk. This type of lesion could therefore incapacitate workers carrying out their daily activities.

Key Words: Musculoskeletal disease, risk factor, human engineering, administrative personnel, Colombia (source: MeSH, NLM).

$\mathrm{U}$ no de los mayores retos de la ergonomía ha sido el estudio de la interacción del hombre frente a los requerimientos físicos (postura, fuerza, movimiento). Cuando estos requerimientos sobrepasan la capacidad de respuesta del individuo o no hay una adecuada recuperación biológica de los tejidos, este esfuerzo puede asociarse con la presencia de lesiones músculo-esqueléticas relacionadas con el trabajo (LME) (1). Actualmente, se reconoce que el mecanismo de aparición de las LME es de naturaleza biomecánica; cuatro teorías explican el mecanismo de aparición: la teoría de la interacción multivariante (factores genéticos, morfológicos, psicosociales y biomecánicos), la teoría diferencial de la fatiga (desequilibrio cinético y cinemático), la teoría cumulativa de la carga (repetición) y finalmente la teoría del esfuerzo excesivo (fuerza) (2).

Las LME son la causa más común de dolores severos de larga duración y de discapacidad física. Los estudios epidemiológicos realizados en diversos países muestran que las LME se presentan en las diversas actividades humanas y en todos los sectores económicos, e implica un inmenso costo para la sociedad (estimado en 215 mil millones de dólares por año, sólo en los Estados Unidos) $(3,4)$. 
En los Estados Unidos, las LME son la primera causa de discapacidad, y suman más de 131 millones de visitas de pacientes a los servicios médicos en el año $(5,6)$. El aumento significativo de la incidencia y de la prevalencia de las LME en el miembro superior es del $60 \%$ en ciertos puestos de trabajo, mientras que la lumbalgia es una sintomatología observable en todos los trabajadores, en toda la población y en todas las categorías profesionales (7).

En Colombia, un estudio realizado por el Instituto de Seguros Sociales en 1997, sobre el síndrome del túnel carpiano en 248 trabajadores de diferente actividad económica, mostró una prevalencia del 20,9 \%, en primer lugar en el sector de alimentos, seguido por el sector de las flores (8). En un estudio del perfil epidemiológico de una Administradora de Riesgos Profesionales en 1998, se encontró que en las empresas de más de 60 trabajadores, el $29 \%$ de ellos estaban sometidos a sobre-esfuerzo y el $51 \%$ a posturas inadecuadas en su labor (9). Se ha estimado que la incidencia de algunas enfermedades ocupacionales, entre las que figuran las LME, presentaron una incidencia de 68063 casos en 1985 y llegaron a los 101645 casos en el 2000 (10).

En una revisión realizada durante los años de 1997 y 2000 en la Unidad de Salud donde son atendidos los administrativos de la Universidad del Cauca, se encontró que la atención en el Servicio de Fisioterapia durante ese período fue de 7397 sesiones y el motivo de remisión fue dolor músculo-esquelético, razón importante para el planteamiento y desarrollo del presente estudio, cuyo propósito fue establecer la frecuencia de las lesiones músculoesqueléticas en trabajadores administrativos y su posible asociación con factores de riesgo ergonómico. Los resultados del estudio nos llevan a recomendar medidas preventivas con el fin de disminuir los trastornos músculoesqueléticos en la población afectada.

\section{MÉTODOS}

El presente estudio de tipo observacional descriptivo de corte transversal se llevó a cabo en la Universidad del Cauca durante el periodo de Julio 2002 a Junio 2003. Se solicitó a la División de Recursos Humanos el listado de los funcionarios administrativos de la Institución, con la información de cargo, dependencia y tiempo de vinculación. Los criterios de selección fueron: tener un tiempo de vinculación igual o mayor a 1 año y ser trabajador de planta en la Institución. De 465 trabajadores de las áreas administrativas, un total de 145 (31,2 \%) trabajadores fueron reclutados en el estudio por medio de un muestreo aleatorio simple (11). 
Los participantes fueron encuestados con dos instrumentos: 1) un formato para análisis del puesto de trabajo entregado por la Administradora de Riesgos Profesionales, en el que se consignan los aspectos relacionados con la postura, el patrón laboral, la carga de peso, la actividad, el puesto de trabajo, la mesa y la silla de trabajo, y 2) un cuestionario nórdico Kourinka para análisis de síntomas músculo-esqueléticos, en el que se interroga al trabajador sobre la presencia de molestia o de dolor osteomuscular en alguna parte del cuerpo durante los últimos 6 meses (12). Todos los procedimientos de la investigación se realizaron de acuerdo con los principios bioéticos establecidos en la Declaración de Helsinki (13).

Para el procesamiento y análisis estadístico de los datos, se construyó una base de datos en Epi-info 6.04 (Atlanta-Georgia, USA, 1996). Las variables continuas se expresaron con la media \pm desviación estándar y las variables discretas se expresaron en frecuencias y proporciones. Posteriormente, se realizó un análisis bivariado entre la lesión músculo-esquelética y los factores ocupacionales del oficio para determinar la asociación entre la aparición de la LME y los factores de riesgo ergonómico (postura, fuerza, movimiento). Además, para estimar el riesgo relativo, se calculó los OR con intervalo de confianza del $95 \%$ (IC $95 \%$ ).

\section{RESULTADOS}

En la Tabla 1 se indican las características demográficas de la población. Del total de sujetos estudiados, el 59,3 \% correspondió al sexo femenino mientras que el 40,7 \% correspondió al sexo masculino. La edad promedio de los trabajadores fue de 41 años y el promedio de antigüedad en el cargo fue de 10 años. Respecto a la actividad laboral se observó que el 80,6 \% de los administrativos realizaban labores secretariales.

En la Tabla 2, se describen los riesgos ocupacionales del oficio. Aquí se observó con mayor frecuencia que los trabajadores realizaban movimientos repetitivos, utilizaban la posición sentada. Los patrones de trabajo más utilizados eran transportar, alcanzar y subir objetos. Al realizar los movimientos, referían tensión muscular continua durante la actividad laboral.

Como se indica en la Tabla 3, las LME más frecuentes en orden de aparición fueron dolor en la zona baja de la espalda, dolor en la zona alta de la espalda, dolor de cuello, dolor en hombros y dolor en muñecas y manos. El estudio reporto que el 56,5 \% (82/145) de los trabajadores administrativos presentaron sintomatología dolorosa de los cuales el 70,3 \% (101/145) pertenecían al sexo femenino, con un promedio de edad de 40,4 años y una an- 
tigüedad promedio en el cargo de 9,1 años. Para los hombres que presentaron sintomatología dolorosa (44/145), la media de edad fue de 42 años y la antigüedad promedio en el cargo fue de 11,3 años. Al comparar estas variables, no se encontraron diferencias significativas entre ambos géneros (datos no mostrados).

Tabla 1. Características sociodemográficas

\begin{tabular}{lc}
\hline \multicolumn{1}{c}{ Características } & $(\%)$ \\
\hline Edad (años) & $41,1 \pm 8,3$ \\
Media \pm DS & $25-59$ \\
Rango & $86(59,3)$ \\
Sexo & $59(40,7)$ \\
Femenino & \\
Masculino & $10,0 \pm 5,7$ \\
Antigüedad en el cargo & $1-32$ \\
Media \pm DS & \\
Rango & $117(80,6)$ \\
Actividad laboral & $5(3,4)$ \\
Secretaria & $5(3,4)$ \\
Cajera & $4(2,7)$ \\
Enfermera & $4(2,7)$ \\
Vigilante & $3(2,1)$ \\
Jefe de división & $2(1,4)$ \\
Instructor & $2(1,4)$ \\
Medico & $2(1,4)$ \\
Odontólogo & $1(0,7)$ \\
Aseador & \\
Ingeniero &
\end{tabular}

Para establecer la asociación de algunos factores de riesgo ergonómico con la aparición de LME, se tuvo en cuenta las LME mas frecuentemente reportadas en la población estudio (cuello, zona alta de la espalda, zona baja de la espalda); sin embargo, es necesario aclarar que el análisis de riesgo se realizó para todas las molestias osteomusculares reportadas, pero no se encontraron asociaciones estadísticamente significativas.

En la Tabla 4 se puede apreciar la asociación de factores de riesgo ergonómico con la aparición de LME, donde, la asociación más significativa se observa en los trabajadores que refieren tensión muscular con la presencia de LME en cuello. 
322 REVISTA DE SALUD PÚBLICA • Volumen 7 (3), Noviembre 2005

Tabla 2. Características ocupacionales

\begin{tabular}{|c|c|c|c|}
\hline Características & $\begin{array}{c}\mathrm{Si} \\
\mathrm{n}(\%)\end{array}$ & $\begin{array}{c}\text { No } \\
\text { n (\%) }\end{array}$ & $\begin{array}{c}\text { No aplica } \\
\mathrm{n}(\%)\end{array}$ \\
\hline \multicolumn{4}{|l|}{ Tipo de Trabajo } \\
\hline Solo & $71(49,0)$ & $74(51,0)$ & \\
\hline En equipo & $89(61,4)$ & $56(38,6)$ & \\
\hline Variado & $83(57,3)$ & $62(42,7)$ & \\
\hline Repetitivo & $75(51,8)$ & $70(48,2)$ & \\
\hline \multicolumn{4}{|l|}{ Postura } \\
\hline De pie & $52(35,9)$ & $93(64,1)$ & \\
\hline Sentado & $115(79,3)$ & $30(20,7)$ & \\
\hline Inclinado & $49(33,8)$ & $96(66,2)$ & \\
\hline Caminando & $67(46,2)$ & $78(53,8)$ & \\
\hline \multicolumn{4}{|l|}{ Patrón } \\
\hline Transportar & $48(33,1)$ & $29(20,0)$ & $68(46,9)$ \\
\hline Alcanzar & $47(32,4)$ & $29(20,0)$ & $69(47,6)$ \\
\hline Subir & $38(26,2)$ & $38(26,2)$ & $69(47,6)$ \\
\hline Empujar & $28(19,3)$ & $45(31,0)$ & $72(49,7)$ \\
\hline Arrastrar & $27(18,6)$ & $41(28,3)$ & $77(53,1)$ \\
\hline \multicolumn{4}{|l|}{ Manejo de Cargas } \\
\hline De manera adecuada & $19(13,1)$ & $25(17,2)$ & $101(69,7)$ \\
\hline Sobre limites permisibles & $25(17,2)$ & $12(8,3)$ & $108(74,5)$ \\
\hline A largas distancias & $8(5,5)$ & $29(20,0)$ & $108(74,5)$ \\
\hline Toda la jornada & $17(11,7)$ & $17(11,7)$ & $111(76,6)$ \\
\hline \multicolumn{4}{|l|}{ Movimientos } \\
\hline Indebidos & $57(39,3)$ & $88(60,7)$ & \\
\hline Tensión Muscular & $103(71,0)$ & $42(29,0)$ & \\
\hline \multicolumn{4}{|l|}{ Puesto de trabajo } \\
\hline Presencia de obstáculos & $28(19,3)$ & $117(80,7)$ & \\
\hline Área suficiente de trabajo & $104(71,7)$ & $41(28,3)$ & \\
\hline Adecuada ubicación de objetos & $78(53,8)$ & $67(46,2)$ & \\
\hline \multicolumn{4}{|l|}{ Mesa y silla de trabajo } \\
\hline Correcta altura mesa vs. silla & $68(46,9)$ & $56(38,6)$ & $21(14,5)$ \\
\hline Correcta altura silla vs. Controles & $65(44,8)$ & $56(38,6)$ & $24(16,6)$ \\
\hline Correcto diseño de silla & $65(44,8)$ & $46(31,7)$ & $34(23,4)$ \\
\hline
\end{tabular}

Tabla 3. Frecuencia de molestias osteomusculares

\begin{tabular}{lcc}
\hline \multicolumn{1}{c}{ Variable } & $\begin{array}{c}\mathrm{Si} \\
\mathrm{n}(\%)\end{array}$ & $\begin{array}{c}\text { No } \\
\mathrm{n}(\%)\end{array}$ \\
\hline Dolor osteomuscular & $82(56,5)$ & $63(43,4)$ \\
Cuello & $71(49,0)$ & $74(51,0)$ \\
\hline Hombros & $69(47,6)$ & $76(52,4)$ \\
\hline Codos & $13(9,0)$ & $132(91,0)$ \\
Muñecas-manos & $44(30,3)$ & $101(69,7)$ \\
\hline Zona alta de la espalda & $77(53,1)$ & $68(46,9)$ \\
Zona baja de la espalda & $82(56,6)$ & $63(43,4)$ \\
\hline Caderas-nalgas-muslos & $45(31,0)$ & $100(69,0)$ \\
\hline Rodillas & $37(25,5)$ & $108(74,5)$ \\
Tobillos-pies & $36(24,8)$ & $109(75,2)$ \\
\hline
\end{tabular}


Tabla 4. Asociación de factores de riesgo ergonómicos con la Aparición de LME

\begin{tabular}{lccc}
\hline \multicolumn{3}{c}{ Aparicion de LME } \\
Factor de Riesgo & \multicolumn{2}{c}{ OR (IC 95 \%) } \\
\cline { 2 - 4 } & Cuello & Zona Alta Espalda & $\begin{array}{c}\text { Zona Baja } \\
\text { Espalda }\end{array}$ \\
\cline { 2 - 4 } Tipo de trabajo & & & \\
Solo & $1,2(0,6-2,4)$ & $1,1(0,6-2,2)$ & $1,7(0,8-3,3)$ \\
En equipo & $1,1(0,6-2,3)$ & $0,8(0,4-1,6)$ & $0,6(0,3-1,3)$ \\
Variado & $0,6(0,3-1,2)$ & $0,8(0,4-1,7)$ & $1,4(0,7-2,7)$ \\
Repetitivo & $1,2(0,6-2,4)$ & $1,5(0,8-3,0)$ & $0,8(0,4-1,6)$ \\
Patrón de trabajo & & & $1,2(0,8-1,8)$ \\
Transportar & $0,7(0,5-1,0)$ & $0,8(0,5-1,2)$ & $1,5(0,9-2,3)$ \\
Alcanzar & $1,0(0,7-1,6)$ & $1,7(1,0-2,8)$ & $1,7(0,8-3,5)$ \\
Postura & & & $0,4(0,2-1,1)$ \\
De pie & $0,8(0,4-1,6)$ & $1,3(0,6-2,6)$ & $3,0(1,4-6,3)$ \\
Sentado & $2,2(0,9-5,2)$ & $1,3(0,6-3,0)$ & $2,8(1,4-5,7)$ \\
Inclinado & $1,1(0,5-2,2)$ & $1,1(0,5-2,2)$ & $1,5(0,7-3,0)$ \\
Caminando & $0,6(0,3-1,2)$ & $0,8(0,4-1,6)$ & $2,8(1,3-6,0)$ \\
Movimientos & & & \\
Indebidos & $2,9(1,4-5,8)$ & $1,3(0,7-2,6)$ & $0,5(0,3-0,8)$ \\
Tensión muscular & $4,6(2,0-10,4)$ & $3,6(1,7-7,8)$ & $1,2(0,9-1,7)$ \\
Mesa y silla de trabajo & $0,7(0,5-1,0)$ & $0,7(0,5-1,0)$ & \\
Correcta altura mesa & $0,7(0,5-1,1)$ & $0,8(0,5-1,1)$ & $0,2-0,6)$ \\
Correcta altura silla & $0,8(0,5-1,2)$ & $0,8(0,5-1,2)$ & \\
Correcto diseño silla & &
\end{tabular}

\section{DISCUSIÓN}

Dentro de los principales factores de riesgo ergonómico asociados a las LME se encuentran: las posturas forzosas, los movimientos repetitivos y los esfuerzos musculares determinados por las acciones de manipulación de carga y movimientos forzosos durante la actividad laboral (14-16). Como limitante del estudio fue la falta de disposición de los jefes de secciones para aplicar los instrumentos al total de la población por lo que fue necesario realizar un muestreo entre los trabajadores que cumplían con los criterios de elegibilidad.

Los resultados del presente estudio muestran que la población, estuvo conformada en su mayoría por mujeres en edad productiva, quienes desempeñan labores secretariales, ocupación en la cual se realizan movimientos repetitivos de las muñecas y de los dedos, acompañados de contracción estática en los músculos de cuello y espalda, que se transforman con el pasar de los años en tensión muscular, incrementando el riesgo de presentar LME como se observa en nuestro estudio.

Cabe mencionar que similar a lo reportado, en otros estudios (17) la postura, la fuerza y el movimiento se consideran factores de riesgo para la apa- 
rición de las LME en la espalda. Adicionalmente, estudios realizados por Armstong (18), Ekberg (19) y Vieira (20), revelan que más del 70 \% de las personas que trabajaban frente a un computador sufren dolores y molestias frecuentes en la espalda, datos no muy alejados de los resultados de nuestra investigación si se tiene en cuenta que en las labores secretariales es frecuente el uso de estos equipos. Nuestro estudio encontró que las LME mas frecuentes en orden de importancia fueron: Zona baja de la espalda, Zona alta de la espalda, cuello, hombros, caderas-nalgas-muslos, muñecas-manos, rodillas, pies y codos. Estudios como el de Juul-Kristensen (21) reportaron que las LME se presentan con mayor frecuencia en la zona baja de la espalda en los trabajadores que utilizan como herramienta de trabajo el computador y deben realizar movimientos repetitivos. Otros estudios han mostrado evidencia a favor de que el trabajo repetitivo se relaciona con LME en cuello y espalda, nuestro estudio reporto una marcada asociación estadística entre tensión muscular y dolor de cuello $(22,23)$.

Con respecto a los factores de riesgo asociados en el presente estudio se observo que alcanzar objetos, mantener posturas forzadas de inclinación y referir tensión muscular durante la actividad laboral significan mayor riesgo.

En conclusión, los resultados del estudio permiten evidenciar que la aparición de las LME esta asociada a factores de riesgo ergonómico (postura, fuerza, y movimiento) contribuyendo a la evidencia científica planteada por Kumar sobre la presunción de que todas las LME ocupacionales son de origen biomecánico (24). Lo anterior justifica la implementación de un programa de vigilancia epidemiológica de la patología músculo-esquelética para prevenir reducción en la productividad laboral, pérdida de tiempo del trabajo, incapacidad temporal o permanente e inhabilidad para realizar las tareas ocupacionales del oficio sumado a un incremento en los costos de compensación al trabajador. Se recomienda para otras investigaciones incluir factores de riesgo comportamentales como tabaquismo, actividad física, antropometría y factores psicosociales, constitución morfológica, entre otros, que permitan establecer cómo éstos pueden afectar el riesgo de padecer LME en las poblaciones Universitarias •

Agradecimientos. Al personal administrativo de la Universidad del Cauca, a la Terapista Ocupacional Sandra Patricia Rivera por su colaboración y apoyo durante la ejecución de este proyecto, a la Fisioterapeuta Gladis Leal Bernal por sus aportes académicos, a la Magister Alice Pouget de Rodríguez por su significativa colaboración en Español y Literatura y a la Fisioterapeuta Angélica Patricia Chamorro Arrieta. 


\section{REFERENCIAS}

1. Miroljub Grozdanovic. Human activity and musculoskeletal injuries and disorders. Medicine and Biology. 2002; 9(2):150-156.

2. Kumar S. Theories of muskuloskeletal injury causation. Ergonomics. 2001;44(1):17-47.

3. Weil D. Valuing the economic consequences of work injury and illness: a comparison of methods and findings. American Journal of Industrial Medicine. 2001;40(4):418-437.

4. Takala J. Global estimates of fatal occupational accidents. Epidemiology. 1999;10(5):645-646.

5. Wegman DH. The potential impact of epidemiology on the prevention of occupational disease. American Journal of Public Health. 1992;82(7):944-954.

6. Pruss A, Corvalan CF, Pastides H, de Hollander AEM. Methodologic considerations in estimating burden of disease from environmental risk factors at national and global levels. International Journal of Occupational and Environmental Health. 2001;7(1):58-67.

7. INSHT. Guía técnica para la evaluación y prevención de los riesgos relativos a la manipulación manual de cargas. Prevención, trabajo y salud. Revista del Instituto Nacional de Seguridad e Higiene en el Trabajo. 2004;2:31-33.

8. Instituto de Seguros Sociales (ISS). Síndrome de túnel del carpo, prevalencia de la enfermedad en poblaciones ocupacionalmente expuestas en Bogotá, Administradora de Riesgos Profesionales. Bogotá, DC; 1999.

9. Colmena Riesgos Profesionales. Programa de vigilancia epidemiológica para la prevención y manejo del dolor lumbar. Bogotá, DC; 1988.

10. Idrovo AJ. Estimación de la incidencia de enfermedades ocupacionales en Colombia 1985-2000. Revista de Salud Pública. 2003;5(3):263-71.

11. R. Polit D, Hungler P. Investigación científica en ciencias de la salud. México: Mc Graw Hill; 2000. p 274-276.

12. Kuorinka I, Jonsson B, Kilbom A, Vinterberg H, Biering-Sorensen F, Andersson G, Jorgensen K. Standardised Nordic questionnaires for the analysis of musculoskeletal symptoms. Applied Ergonomics. 1987;18(3):233-237.

13. The Helsinki Declaration. Orvosi hetilap. 1965;106(36):1715-1716

14. Markowitz SB, Fischer E, Fahs MC, Shapiro J, Landrigan PJ. Occupational disease in New York state: A comprehensive examination. American Journal of Industrial Medicine. 1989;16(4):417-435.

15. INSHT. Guía técnica para la evaluación y prevención de los riesgos relativos a la manipulación manual de cargas. Madrid; 1997.

16. Nayha S, Videman T, Laakso M, Hassi J. Prevalence of low back pain and other musculoeskeletal symptoms and their association with work in finish reindeer herders. Scandinavian Journal of Rheumatology. 1991;20(6):406-413.

17. Hildebrandt, VA. A review of epidemiological research on risk factors of lowback pain, In: Buckl P. (ed), Musculoskeletal Disorders at work, Taylor and Francis, 1997. p 9-16.

18. Armstong TJ, Bucke P, Fine LJ, Hagberg M, Jonsson B, Kilbom A, et al. A conceptual model for work-related neck and upper-limb musculoskeletal disor- 
ders. Scand Journal of Work, Environment and Health. 1993;19(2):7384.15.

19. Ekberg K, Bjorkqvist, B, Malm P, Bjerre-Kiely B, Karlsson M, Axlson O. Casecontrol study of risk factors for disease in the neck and shoulder area. Occupational and Environmental Medicine. 1994;51(4):262-266.

20. Vieira ER, Kumar S. Working postures: a literature review. Journal of Occupational Rehabilitation. 2004;14(2):143-59.

21. Juul-Kristensen B, Jensen C. Self reported workplace related ergonomic conditions as prognostic factors for musculoskeletal symptoms: The "BIT" follow up study on office workers. Occupational Medicine. 2005;62:188-194.

22. Jensen C. Development of neck and hand-wrist symptoms in relation to duration of computer use at work. Scandinavian Journal of Work, Environment and Health. 2003;29:197-205.

23. Feuerstein M, Nicholas RA, Huang GD, Haufler AJ, Pransky G, Robertson M. Workstyle: development of a measure of response to work in those with upper extremity pain. Journal of Occupational Rehabilitation. 2005;15(2):87-104.

24. Kumar S. Selected theories of musculoskeletal injury causation. In: Kumar S. (ed), Biomechanics in Ergonomics. Philadelphia: CRC Press; 1999. p 3-24. 